Canad. Math. Bull. Vol. 19 (4), 1976

\title{
SOME ARITHMETIC PROPERTIES OF A SPECIAL SEQUENCE OF INTEGERS
}

\author{
BY \\ L. CARLITZ
}

1. Leeming [3] has defined a sequence of polynomials $\left\{Q_{4 n}(x)\right\}$ and a sequence of integers $\left\{Q_{4 n}\right\}$ by means of

$$
\frac{\cosh x t+\cos x y}{\cosh t+\cos t}=\sum_{n=0}^{\infty} Q_{4 n}(x) \frac{t^{4 n}}{(4 n) !}
$$

and

$$
Q_{4 n}=Q_{4 n}(0)
$$

Thus

$$
\frac{2}{\cosh t+\cos t}=\sum_{n=0}^{\infty} Q_{4 n} \frac{t^{2 n}}{(4 n) !}
$$

and equivalently

$$
\sum_{k=0}^{n}\left(\begin{array}{l}
4 n \\
4 k
\end{array}\right) Q_{4 k}=0 \quad(n>0)
$$

Moreover

$$
(-4)^{n} Q_{4 n}=\sum_{k=0}^{2 n}(-1)^{k}\left(\begin{array}{c}
4 n \\
2 k
\end{array}\right) E_{2 k} E_{4 n-2 k}
$$

where the $E_{2 k}$ are the Euler numbers defined by [5, Ch. 2]

$$
\frac{1}{\cosh t}=\sum_{n=0}^{\infty} E_{2 n} \frac{t^{2 n}}{(2 n) !} \text {. }
$$

Leeming shows also that the $Q_{4 n}$ are all odd and that

$$
(-1)^{n} Q_{4 n}>0 \quad(n=0,1,2, \ldots) .
$$

It is well known (see for example [4, Ch. 14]) that the Euler numbers satisfy Kummer's congruence:

$$
\sum_{s=0}^{r}(-1)^{s}\left(\begin{array}{l}
r \\
s
\end{array}\right) E_{2 n+s(p-1)} \equiv 0\left(\bmod p^{r}\right) \quad(2 n \geq r),
$$

Received by the editors June 10, 1976.

Supported in part by NSF grant GP-37924X. 
where $p$ is an arbitrary odd prime. For $p=2$, Frobenius [2, p. 477] stated the following result: If $a \geq 0, b>0, r>0$, then the power of 2 dividing the number

$$
\sum_{s=0}^{r}(-1)^{s}\left(\begin{array}{l}
r \\
s
\end{array}\right) E_{2 a+2 s b}
$$

is the same as the power of 2 dividing the number $(2 b)^{r} r$ !. For proof see [1]. Frobenius [2, p. 477] proved that

$$
E_{2 n} \equiv 1-2 n+8\left(\begin{array}{l}
n \\
2
\end{array}\right)(\bmod 16)
$$

as well as more precise results.

In the present note we shall prove first the following result corresponding to (1.8). Let $p$ be an odd prime and let $m \geq 0$. Then

$$
\sum_{s=0}^{r}(-1)^{r-s}\left(\begin{array}{l}
r \\
s
\end{array}\right) Q_{4 n+s m} \equiv 0\left(\bmod p^{r}\right) \quad(4 n \geq r)
$$

where $m$ is divisible by $p-1$ or $p^{2}-1$ according as $p \equiv 1$ or $3(\bmod 4)$. In particular

$$
\begin{aligned}
& \text { (1.11) } \sum_{s=0}^{r}(-1)^{r-s}\left(\begin{array}{l}
r \\
s
\end{array}\right) Q_{4 n+s(p-1)} \equiv 0\left(\bmod p^{r}\right) \quad(4 n \geq r, p \equiv 1(\bmod 4)), \\
& \text { (1.12) } \sum_{s=0}^{r}(-1)^{r-s}\left(\begin{array}{l}
r \\
s
\end{array}\right) Q_{4 n+s\left(p^{2}-1\right)} \equiv 0\left(\bmod p^{r}\right) \quad(4 n \geq r, p \equiv 3(\bmod 4)) .
\end{aligned}
$$

Also, corresponding to (1.9), we show that

$$
Q_{4 n} \equiv 1-2 n+8\left(\begin{array}{l}
n \\
2
\end{array}\right) \quad(\bmod 16)
$$

in particular

$$
Q_{4 n} \equiv(-1)^{n}(\bmod 4), \quad Q_{4 n} \equiv 1-2 n(\bmod 8)
$$

Note that, by (1.9) and (1.13),

$$
Q_{4 n} \equiv E_{2 n}(\bmod 16)
$$

Thus it would be of interest to know whether $Q_{4 n}-E_{2 n}$ is divisible by some high power of 2 . 
2. Proof of (1.10). We have

$$
\begin{aligned}
\frac{2}{\cosh t+\cos t}= & \frac{4}{e^{t}+e^{-t}+e^{i t}+e^{-i t}} \\
= & \frac{4}{4-\left(1-e^{t}\right)-\left(1-e^{-t}\right)-\left(1-e^{i t}\right)-\left(1-e^{-i t}\right)} \\
= & \sum_{a, b, c, d=0}^{\infty} 4^{-a-b-c-d}(a, b, c, d)\left(1-e^{t}\right)^{a}\left(1-e^{-t}\right)^{b} \\
& \cdot\left(1-e^{i t}\right)^{c}\left(1-e^{-i t}\right)^{d},
\end{aligned}
$$

where

$$
(a, b, c, d)=\frac{(a+b+c+d) !}{a ! b ! c ! d !}
$$

Expanding (2.1) we get

$\sum_{a, b, c, d=0}^{\infty} 4^{-a-b-c-d}(a, b, c, d) \sum_{a^{\prime}, b^{\prime}, c^{\prime}, d^{\prime}}(-1)^{a^{\prime}+b^{\prime}+c^{\prime}+d^{\prime}}\left(\begin{array}{c}a \\ a^{\prime}\end{array}\right)\left(\begin{array}{c}b \\ b^{\prime}\end{array}\right)\left(\begin{array}{c}c \\ c^{\prime}\end{array}\right)\left(\begin{array}{c}d \\ d^{\prime}\end{array}\right)$

$\cdot e^{\left(a^{\prime}-b^{\prime}+\left(c^{\prime}-d^{\prime}\right) i\right) t}$.

Hence, by (1.3),

$Q_{n}=\sum_{\substack{a, b, c, d \\ a^{\prime}, b^{\prime}, c^{\prime}, d^{\prime}}}(-1)^{a^{\prime}+b^{\prime}+c^{\prime}+d^{\prime}}(a, b, c, d)\left(\begin{array}{c}a \\ a^{\prime}\end{array}\right)\left(\begin{array}{c}b \\ b^{\prime}\end{array}\right)\left(\begin{array}{c}c \\ c^{\prime}\end{array}\right)\left(\begin{array}{c}d \\ d^{\prime}\end{array}\right)$

$$
\cdot\left(a^{\prime}-b^{\prime}+\left(c^{\prime}-d^{\prime}\right) i\right)^{n},
$$

where $Q_{n}=0$ if $n$ is not divisible by 4 . It is clear, by finite differences, that we may assume in (2.2) that $a+b+c+d \leq n$.

It follows from (2.2) that, for arbitrary $m>0$,

$$
\begin{array}{r}
\sum_{s=0}^{r}(-1)^{r-s}\left(\begin{array}{l}
r \\
s
\end{array}\right) Q_{n+s m}=\sum_{\substack{a, b, c, d \\
a^{\prime}, b^{\prime}, c^{\prime}, d}}(-1)^{a^{\prime}+b^{\prime}+c^{\prime}+d^{\prime}}(a, b, c, d)\left(\begin{array}{c}
a \\
a^{\prime}
\end{array}\right)\left(\begin{array}{c}
b \\
b^{\prime}
\end{array}\right)\left(\begin{array}{l}
c \\
c^{\prime}
\end{array}\right)\left(\begin{array}{l}
d \\
d^{\prime}
\end{array}\right) \\
\cdot\left(a^{\prime}-b^{\prime}+\left(c^{\prime}-d^{\prime}\right) i\right)^{n}\left\{\left(a^{\prime}-b^{\prime}+\left(c^{\prime}-d-\right) i\right)^{m}-1\right\}^{r} .
\end{array}
$$

Now if $a+b i$ is an arbitrary Gaussian integer and $p$ is a prime, $p \equiv 1(\bmod 4)$, then

$$
(a+b i)^{p} \equiv a+b i(\bmod p)
$$

however if $p \equiv 3(\bmod 4)$, then

$$
(a+b i)^{p^{2}} \equiv a+b i(\bmod p) .
$$

Therefore we have

$$
\left.\sum_{s=0}^{r}(-1)^{r-s}\left(\begin{array}{l}
r \\
s
\end{array}\right) Q_{n+s m} \equiv 0 \bmod p^{r}\right) \quad(n \geq r)
$$


where $p-1 \mid m$ if $p \equiv 1(\bmod 4)$ while $p^{2}-1 \mid m$ if $p \equiv 3$. This result evidently includes (1.10).

3. Proof of (1.13). We have

$$
1-2 k+8\left(\begin{array}{l}
k \\
2
\end{array}\right)=1-6 k+4 k^{2}=1-5 k+k(4 k-1) .
$$

Put

$$
S_{n}=\sum_{k=0}^{n}\left(\begin{array}{l}
4 n \\
4 k
\end{array}\right)(1-5 k+k(4 k-1)) \text {. }
$$

Then

$$
S_{n}=S_{n}^{\prime}-5 S_{n}^{\prime \prime}+S^{\prime \prime \prime}
$$

where

$$
\begin{aligned}
& S_{n}^{\prime}=\sum_{k=0}^{n}\left(\begin{array}{l}
4 n \\
4 k
\end{array}\right), \\
& S_{n}^{\prime \prime}=\sum_{k=0}^{n} k\left(\begin{array}{l}
4 n \\
4 k
\end{array}\right)=n \sum_{k=1}^{n}\left(\begin{array}{c}
4 n-1 \\
4 k-1
\end{array}\right)=n \sum_{k=0}^{n-1}\left(\begin{array}{c}
4 n-1 \\
4 k
\end{array}\right), \\
& S_{n}^{\prime \prime \prime}=\sum_{k=0}^{n} k(4 k-1)\left(\begin{array}{l}
4 n \\
4 k
\end{array}\right)=n(4 n-1) \sum_{k=1}^{n}\left(\begin{array}{c}
4 n-2 \\
4 k-2
\end{array}\right)=n(4 n-1) \sum_{k=0}^{n-1}\left(\begin{array}{c}
4 n-2 \\
4 k
\end{array}\right) .
\end{aligned}
$$

Since, for $m>0$,

$$
\begin{aligned}
\sum_{4 k \leq m}\left(\begin{array}{c}
m \\
4 k
\end{array}\right) & =\frac{1}{4}\left\{(1+1)^{m}+(1-1)^{m}+(1+i)^{m}+(1-i)^{m}\right\} \\
& =\frac{1}{4}\left\{2^{m}+(1+i)^{m}+(1-i)^{m}\right\},
\end{aligned}
$$

we get

$$
\begin{aligned}
& S_{n}^{\prime}=\frac{1}{4}\left\{2^{4 n}+(-1)^{n} 2^{2 n+1}\right\}, \\
& S_{n}^{\prime \prime}=\frac{1}{4} n\left\{2^{4 n-1}+(-1)^{n} 2^{2 n}\right\}, \\
& S^{\prime \prime \prime}=\frac{1}{4} n(4 n-1) \cdot 2^{4 n-2} .
\end{aligned}
$$

Hence, by (3.2),

(3.3) $S_{n}=2^{4 n-2}-5 n \cdot 2^{4 n-3}+n(4 n-1) \cdot 2^{4 n-4}+(-1)^{n} 2^{2 n-1}-5 n(-1)^{n} 2^{2 n-2}$.

It follows from (3.3) that

$$
S_{n}=0 \quad(n=1,2,3)
$$

and

$$
S_{n} \equiv 0(\bmod 16) \quad(n \geq 4)
$$


so that

$$
S_{n} \equiv 0(\bmod 16) \quad(n=1,2,3, \ldots) .
$$

Therefore by (3.1) and (1.4), we have

$$
Q_{4 n} \equiv 1-2 n+8\left(\begin{array}{l}
n \\
2
\end{array}\right) \quad(\bmod 16) \text {. }
$$

According to the numerical data in [3], the $Q_{4 n}$ have the following residues $(\bmod 16)$ :

\begin{tabular}{|c|c|c|c|c|c|c|c|}
\hline$n$ & 0 & 1 & 2 & 3 & 4 & 5 & 6 \\
\hline$Q_{4 n}$ & 1 & -1 & 5 & 3 & 9 & 7 & -3 \\
\hline
\end{tabular}

These results are evidently in agreement with (3.7).

\section{REFERENCES}

1. L. Carlitz, Kummer's congruences $\left(\bmod 2^{r}\right)$, Monatshefte für Mathematik 63 (1959), 394400.

2. F. G. Frobenius, Uber die Bernoullischen Zahlen und die Eulerschen Polynome, Gesammelte Abhandlungen III, 440-478, Springer, Berlin-Heidelberg-New York, 1968.

3. D. J. Leeming, Some properties of a certain set of interpolation polynomials, Canadian Mathematical Bulletin 18 (1975), 529-537.

4. N. Nielsen, Traité élémentaire des nombres de Bernoulli, Gauthier-Villars, Paris, 1923.

5. N. E. Nörlund, Vorlesungen über Differenzenrechnung, Springer, Berlin, 1924.

DePARTMENT OF MATHEMATICS

DUKE UNIVERSITY

DURHAM, NORTH CAROLINA 27706

U.S.A. 